\title{
COVID-19 in a patient with HIV and Kaposi sarcoma
}

\author{
\begin{tabular}{c} 
Maria-Andrada Corodeanu1, Sorina Vasile1, Anca-Cristiana Oprea1,2 \\
1"Casa Doru" HIV/AIDS Clinical Department, "Dr. Victor Babes" Clinical Hospital of \\
Infectious and Tropical Diseases, Bucharest, Romania \\
2"Carol Davila" University of Medicine and Pharmacy, Bucharest, Romania \\
\hline
\end{tabular}
}

\begin{abstract}
A 53 year old male diagnosed with HIV, SARS-CoV-2 and Kaposi sarcoma developed a purple-brown maculopapular rash on the left calf approximately 6 months before hospitalization and was diagnosed with venous ulcer. The lesions grew in size, spread on the whole body and also appeared on the palate. During this time the patient did not ask for a second opinion and was not monitored by a medical specialist. In December 2020, he developed a severe form of COVID-19 with acute respiratory failure and was admitted to the hospital. He was simultaneously diagnosed with HIV and severe immunosuppression. The skin biopsy confirmed Kaposi sarcoma in the nodular stage. Antiretroviral therapy (ART) was initiated and the patient later received liposomal doxorubicin chemotherapy. The patient slowly recovered whilst showing improvement of his clinical condition and immunological status.
\end{abstract}

Keywords: COVID-19, HIV, Kaposi sarcoma, antiretroviral treatment

\section{INTRODUCTION}

The first SARS-CoV-2 cases were reported in December 2019 in the Wuhan province in China. This disease was named COVID-19 by the World Health Organization starting from February 2020 (1).

According to CDC one of the clinical conditions that could potentially determine the patients to develop severe cases of COVID-19 is HIV infection. The clinical progress of those infected with HIV and SARS-CoV-2, with good immunological status and undetectable viral load, seems to be similar to those without HIV. However, the data we have at this point is inconclusive $(2,3)$.

Kaposi sarcoma (KS) is an angioproliferative multicentric tumor originating in the endothelial cells infected with Human Herpesvirus-8 (HHV-8). KS is classified into 4 types: epidemic, associated with HIV infection, iatrogenic, associated with immunosuppressive treatment, endemic (African) and classic (sporadic). In the epidemic type the lesions usually develop on the skin and mucosae but, in the more advanced forms, they can also affect the viscera. Pulmonary involvement occurs in $80-90 \%$ of patients, usually in those with severe immunosuppression who also associate extensive skin and mucous lesions, but it can also present as the main site of involvement in $15 \%$ of cases $(4,5)$.

\section{AIM}

The purpose of this case report is to highlight the early signs of Kaposi sarcoma and the importance of early diagnosis in HIV infected patients.

\section{CASE PRESENTATION}

A 53 year-old male was seen in the emergency department of this hospital because of dyspnea and severe dry cough. The symptoms began approximately 
one month before admission with fever, chills, chest pain, dyspnea and cough. Because his respiratory symptoms became progressively worse he called an ambulance and was hospitalized at SUUB (Spitalul Universitar de Urgență București) where he has diagnosed with SARS-CoV-2 infection. Other than the respiratory symptoms, the patient also presented multiple purple-brown lesions of different sizes disseminated on his whole body.The first lesions developed on the left calf approximately 6 months before admission. He was evaluated at that point in a dermatology department and was diagnosed with venous ulcer.

During hospitalization at SUUB the patient was diagnosed with HIV. The evaluation of the immunological and virusological status showed severe immunosuppression $(C D 4+\mathrm{T}$ cells $=64 / \mathrm{mm} 3)$ and high viral load (HIV RNA = 40,200 copies/ml).

He developed a severe form of pulmonary disease with acute respiratory failure due to both COVID-19 and Kaposi sarcoma. Computed tomography of the chest revealed extensive ground glass opacity, with involvement of more than $75 \%$ of the lung, and bilateral pleural effusion. Thoracocentesis removed 2300 ml serous-blood-stained fluid with multiple red blood cells and lymphocytes. The patient had a fluctuating clinical progress with repeated episodes of fever and dyspnea. He received treatment according to the COVID-19 guidelines, broad-spectrum antibiotics and oxygen therapy. He underwent a left thoracotomy with the attachment of a draining tube after which he was coupled to an active aspiration system. He was later transferred in this hospital for examination and treatment.

He was single, lived in an urban area in Bucharest. He had sex with men and used condoms intermittently. He drank alcohol in moderation and did not smoke or use illicit drugs.

On examination, the patient appeared ill and agitated. The temperature was 36,5 degrees Celsius, he was of normal weight $(58 \mathrm{~kg}, 170 \mathrm{~cm}, \mathrm{IMC}=20 \mathrm{~kg} /$ $\mathrm{m} 2$ ), his skin was pale and showed multiple purplebrown lesions of nodulo-papular shape, with sizes ranging between 1 and $20 \mathrm{~cm}$, disseminated on the limbs, trunk and right ear (Fig. 1-3); he also had a macular violaceous lesion on the palate (Fig. 4); he presented lower limb peripheral edema and laterocervical adenopaties $(0,5 \mathrm{~cm})$. The blood oxygen saturation was $85 \%$ in ambient air and 96\% after oxygen therapy at a flow of $10 \mathrm{l}$ per minute; he was polypneic
(32 respirations/minute); on auscultation the vesicular murmur was abolished bilaterally in the pulmonary bases, he presented pleural friction rub sounds in the inferior half of the left hemythorax and fine crackles in both pulmonary bases.

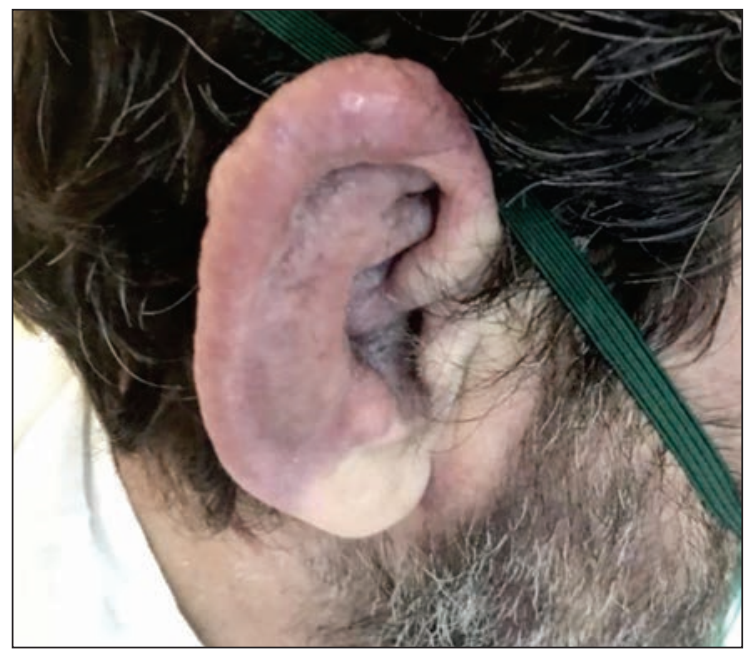

FIGURE 1. Right year lesion
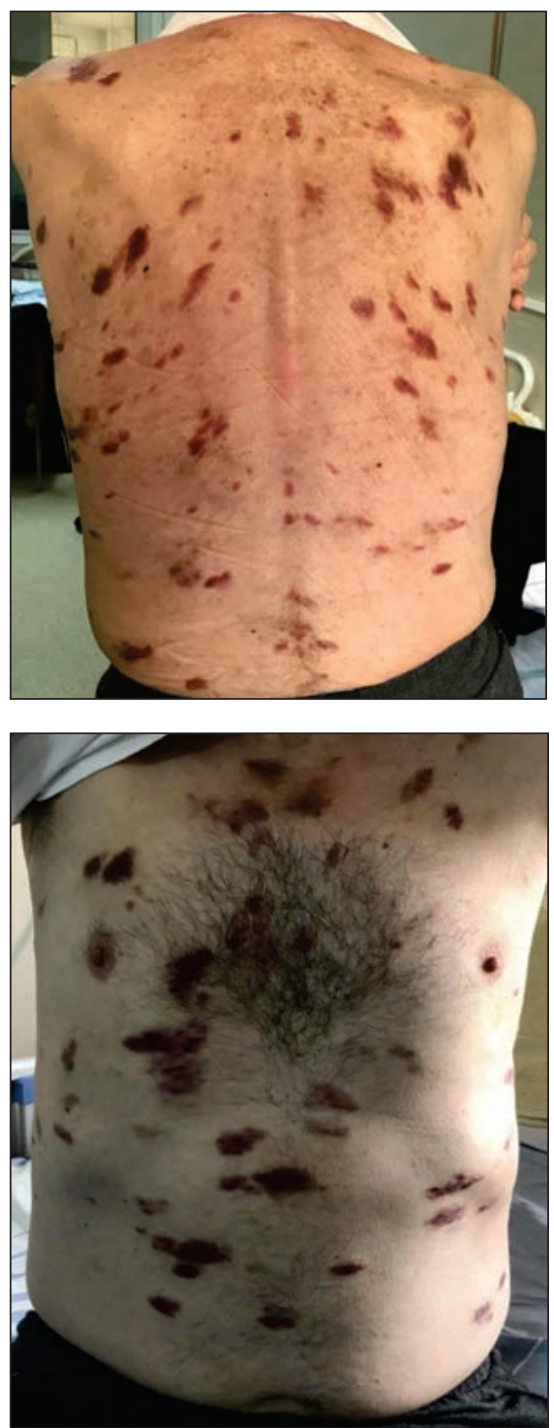

FIGURE 2. Disseminated lesions on the thorax 


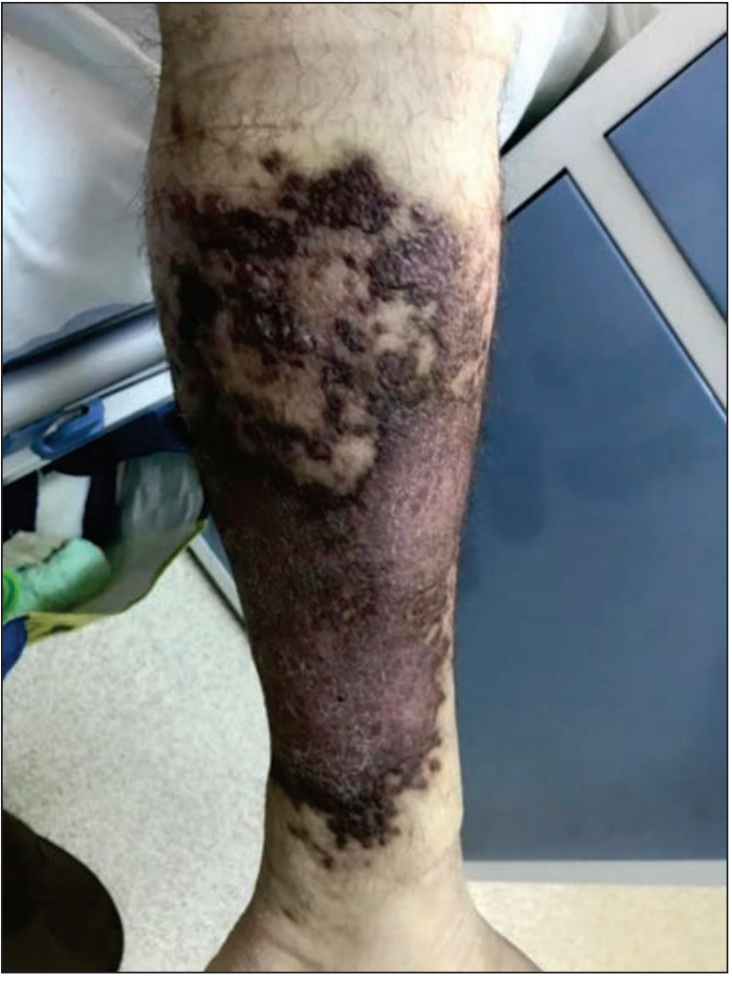

FIGURE 3. Left calf lesion (the primary localization of the tumor)

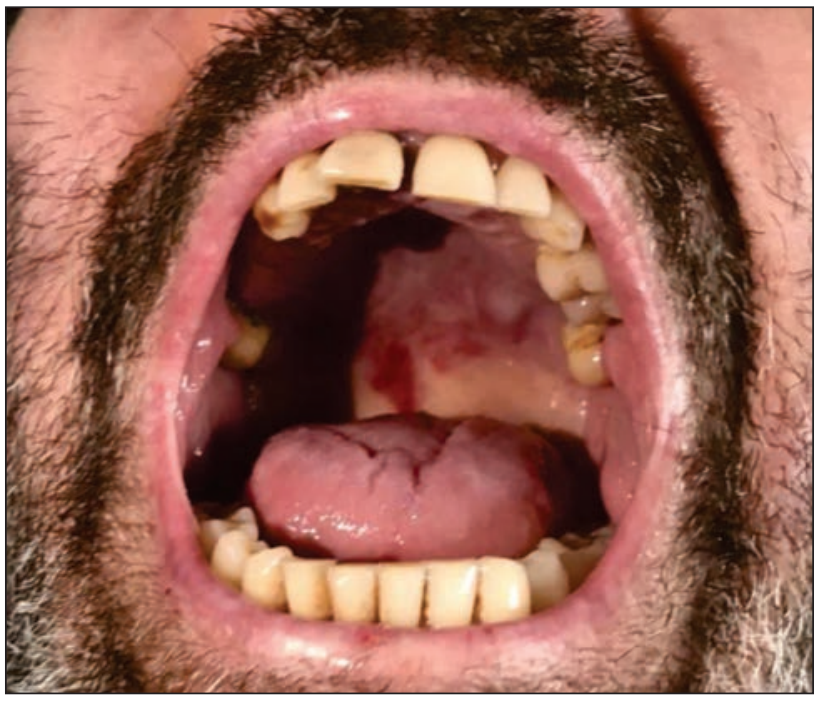

FIGURE 4. Macular violaceous lesions on the oral mucosa, on the palate

The laboratory test results revealed moderate normocytic normochromic anemia, intense inflammatory syndrome, hypoalbuminemia and coagulation abnormalities (Table 1). Microscopic examination of clinical samples from the sputum using the ZiehlNeelsen stain was negative for acid-fast bacilli and the Lowenstein-Jensen sputum cultures (60 days under observation) were negative for Mycobacterium tuberculosis. The serology for hepatitis B and hepatitis $\mathrm{C}$ were negative. The immunological assessment in our clinic revealed severe immunosuppression
$(\mathrm{CD} 4+\mathrm{T}$ cells $=150 / \mathrm{mm} 3 ; \mathrm{CD} 4+/ \mathrm{CD} 8+=0.29)$ and high viral load (HIV RNA = 879,721 copies/ml).

TABLE 1. Laboratory data

\begin{tabular}{|c|c|}
\hline Parameter & Value \\
\hline Leukocytes & $8500 / \mu \mathrm{l}$ \\
\hline Lymphocytes & $1200 / \mu \mathrm{l}$ \\
\hline Neutrophils & $6500 / \mu \mathrm{l}$ \\
\hline Thrombocytes & $303000 / \mu \mathrm{l}$ \\
\hline Hemoglobin & $9,5 \mathrm{~g} / \mathrm{dl} \downarrow$ \\
\hline $\mathrm{C}$ reactive protein & $10.3 \mathrm{mg} / \mathrm{dl} \uparrow$ \\
\hline ESR & $46 \mathrm{~mm} / \mathrm{h} \uparrow$ \\
\hline Procalcitonin & $0.07 \mathrm{ng} / \mathrm{ml}$ \\
\hline Feritin & $387.2 \mathrm{ng} / \mathrm{ml} \uparrow$ \\
\hline IL-6 & $35.78 \mathrm{pg} / \mathrm{ml} \uparrow$ \\
\hline Fibrinogen & $616 \mathrm{mg} / \mathrm{dl} \uparrow$ \\
\hline D-dimers & $3.57 \mu \mathrm{g} / \mathrm{ml} \uparrow$ \\
\hline Albumin & $22 \mathrm{~g} / \mathrm{dl} \downarrow$ \\
\hline $\mathrm{LDH}$ & $152 \mathrm{U} / \mathrm{I}$ \\
\hline Creatinine & $0.8 \mathrm{mg} / \mathrm{dl}$ \\
\hline TGP & $32 \mathrm{U} / \mathrm{I}$ \\
\hline TGO & $15 \mathrm{U} / \mathrm{I}$ \\
\hline GGT & $27 \mathrm{U} / \mathrm{I}$ \\
\hline PT & $84 \%$ \\
\hline Glucose & $108 \mathrm{mg} / \mathrm{dl}$ \\
\hline $\mathrm{AgHBs}$ & negative \\
\hline HCV antibodies & negative \\
\hline $\mathrm{CD} 4+$ & 150 cells \\
\hline CD4/CD8 & 0.29 \\
\hline HIV RNA & 879721 copies/ml \\
\hline Ziehl-Neelson AFB stain & AFB negative \\
\hline $\begin{array}{l}\text { Lowenstein-Jensen cultures for } \\
\text { M. tuberculosis ( } 60 \text { days under } \\
\text { observation) }\end{array}$ & negative \\
\hline Urinalysis & normal \\
\hline Urine culture & negative \\
\hline
\end{tabular}

The imaging studies of the chest revealed: bilateral pleural effusion, free-flowing and partially loculated, small on the left side and medium on the right side; mixed reticular and nodular opacities in the inferior $2 / 3$ of the left lung and in the right base; thickened septal lines and the partial collapse of the right lung due to the pleural fluid (Figures 5, 6).

The pleural fluid removed through thoracocentesis was cloudy, with exudate characteristics (Rivalta +++ , total proteins $=3,1 \mathrm{~g} / \mathrm{dl}$; fluid $\mathrm{TP} /$ serum $=0.55$, $\mathrm{ADA}=75.5 \mathrm{U} / \mathrm{l}, \mathrm{LDH}=357 \mathrm{U} / \mathrm{l}$; fluid LDH /serum = 2.20, glucose $=50 \mathrm{mg} / \mathrm{dl}$, pleural colesterol $=47 \mathrm{mg} /$ dl); with 97\% mononuclear cells, 3\% PMNs, frequent erythrocytes and atypical cells, negative AFB smear, negative Lowenstein-Jensen cultures (after 60 days).

The skin biopsy showed dermal fusocellular proliferation of fusiform cells displayed in fascicular po- 


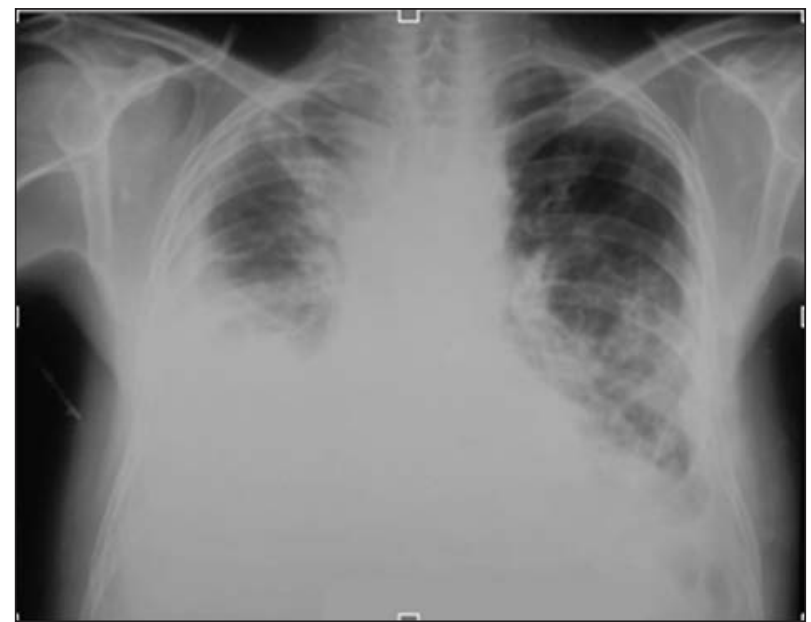

FIGURE 5. Cardio-pulmonary radiography: bilateral pleural effusion and mixed infiltrates in the inferior $2 / 3$ of the left lung and the right base

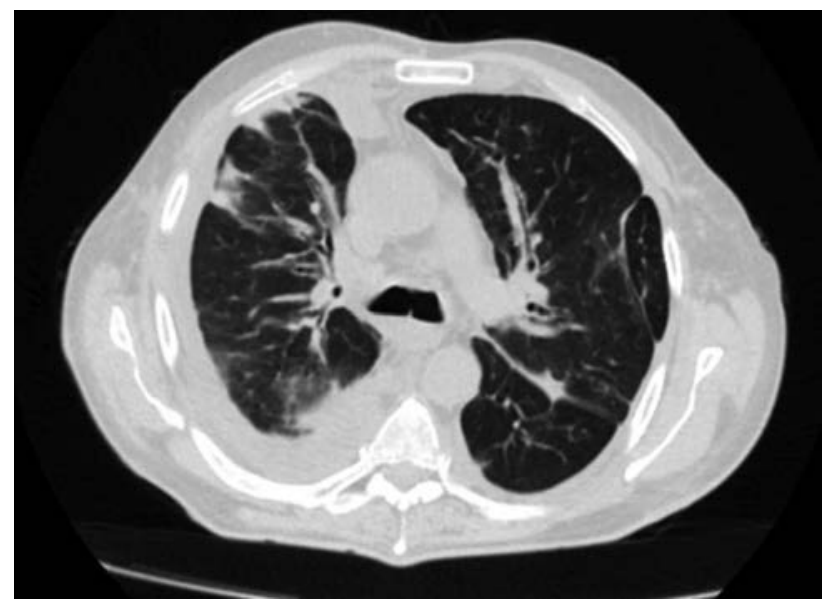

FIGURE 6. Pulmonary CT: bilateral pleural effusion; thickening of the intra- and inter- septa; the collapse of the right lung

sitions, with eosinophilic cytoplasm, irregular margins and vesicular nuclei, rare mitosis, frequent intracytoplasmic spaces; numerous vesicular spaces of small sizes of flattened endothelial cells containing red blood cells; frequent hematic extravasation, frequent capillary vessels and vascular ectasia, small hemosiderin deposits and minimal lymphocyte infiltration. It confirmed the diagnosis of Kaposi sarcoma (nodular stage).

\section{Differential diagnosis}

With regard to the pulmonary affliction, seeing as the patient was severely immunosuppressed, with HIV infection and an AIDS defining illness, we firstly considered other infectious causes. Pulmonary tuberculosis can develop in HIV infected patients 100 times more frequently than in the normal population. The clinical signs and symptoms can vary depending on the number of CD4+ T cells. Immunosuppressed patients usually develop atypical or paucisymptomatic tuberculosis, frequently with remote locations, seeing as the typical symptoms of TB become apparent because of the host's immune response. Those afflicted, with an acceptable immunological status, can develop typical forms of TB, whereas those with severe immunosuppression, with a CD4+ count under 200/mm3, could potentially only display adenopathies as the singular clinical sign with normal or atypical imaging chest studies. Tuberculosis was disproved after the analysis of the cultures and smears from the sputum and pleural fluid (4).

Another possible etiology is Pneumocystis jirovecii pneumonia. The clinical symptoms and the imaging studies can be similar with those in COVID-19. The ground glass opacity on CT scans can be present in both. One difference between the two is the location of the lesions. SARS-CoV-2 has been showed to affect predominantly the periphery of the pulmonary fields whilst in pneumocystosis the lesions are mainly dispersed in the central region. This disease was ruled out since there were no characteristic cysts found in bronchoalveolar lavage. Other bacterial or fungal pathologies were ruled out after the analysis of the sputum and pleural fluid $(1,4,6)$.

Other non-infectious causes of pulmonary disease (other types of cancer, pulmonary thrombo-embolism, collagen afflictions etc.) were excluded with he help of the imaging and laboratory studies.

We considered Castleman's disease and acroangiodermatitis for the differential diagnosis of the skin lesions. Castleman's disease is a lymphoproliferative disorder associated with HHV-8 infection that can appear in HIV infected patients. In the multicentric type the skin lesions usually appear as hyperpigmentated plaques. The skin biopsy wan not evocative of this disease. Acroangiodermatitis is a rare benign angioproliferative disease where purple-brown nodules or plaques appear on the inferior limbs usually in association with chronic venous disease. As opposed to the lesions in KS, they are painful, itching, with a tendency for bleeding and ulceration and are not expanding in nature $(7,8)$.

\section{Final diagnosis}

The laboratory and imaging studies established the diagnoses of interstitial and alveolar pneumonia with acute respiratory failure of mixed origin, severe 
SARS-CoV-2 infection, disseminated Kaposi sarcoma (with mucosal, cutaneous and visceral involvement), bilateral serous hemorrhagic pleurisy (due to KS) and HIV-AIDS infection with severe immunosuppression (C3 stage), moderate normocytic normochromic anemia and secondary hypoalbuminemia.

\section{Therapeutic focus and assessment}

The treatment for COVID-19 was administered in accordance with the national guidelines. He received oxygen therapy (with a maximum flow of 10L per minute), corticotherapy with intravenous dexametasone, prophylaxis for thrombo-embolic incidents with subcutaneous enoxaparin. Also, given the extension and appearance of the pulmonary lesions and the fact that the patient was severely immunosuppressed, a bacterial suprainfection could not have been excluded at first; therefore, he received wide spectrum antibiotic therapy with intravenous meropenem until the the results of the cultures were done. Prophylaxis for Pneumocystis jirovecii pneumonia with Trimethoprim/sulfamethoxazole was initiated. He also received bronchodilatators, antitussives, antihistamines, anxiolytics, pain-killers, diuretics and albumin.

Regarding the HIV infection, initiation of combined antiretroviral therapy is the main method of treatment, that could potentially lead to the clinical regression of the KS lesions and slow down the progress of the disease. Some patients with severe symptoms, visceral involvement and rapid dissemination are also indicated to receive systemic chemotherapy. The drug of choice in those situation is liposomal doxorubicin (in the absence of cardiovascular contraindications) (5).

Antiretroviral therapy was initiated in accordance with the EACS guidelines with an integrase inhibitor - Ralegravir in doses of $400 \mathrm{mg}$ - 2 times/day, and 2 reverse-transcriptase inhibitors - Tenofocir and Emtricitabine in doses of $200 \mathrm{mg} / 245 \mathrm{mg}-1$ time/ day. The patient was later seen in an oncology department where he was administered chemotherapy with liposomal doxorubicin (Caelix).

The patient's clinical progress, initially unfavourable, was ameliorated. The respiratory insufficiency and the inflammatory syndrome disappeared, the skin lesions showed signs of regression and the cellular immunity improved (CD4+ T cells $=396 / \mathrm{mmc}$ ). He has discharged after one month of hospitalization with a good clinical status, without need oxygen therapy, with good cardio-respiratory and digestive functions, with the recommendation of continuation of the antiretroviral and cytostatic drugs.

\section{DISCUSSION}

Epidemic Kaposi sarcoma (KS) associated with HIV infection is the most common form of KS. The induction of antiretroviral therapy lowered the incidence of the disease and diminished the mortality rates in the whole world, although they remain high in some regions (such as sub-saharan Africa). The progression rate of the disease is usually fast, leading to visceral dissemination and, in the cases with the worst clinical outcomes, the involvement of the respiratory or digestive systems $(9,10)$.

Studies showed that approximately $10-20 \%$ of the patients diagnosed with Kaposi sarcoma are identified with advanced forms of disease and that a percent of 6,6\% develop IRIS (immune reconstitution inflammatory syndrome). IRIS develops after the initiation of effective antiretroviral therapy, along with the amelioration of the host's immune status and the reduction of the viral load, revealing itself through the paradoxically worsening of the patient's previous infections $(4,11)$.

The available data regarding the risks of SARSCoV-2 infection in patients with HIV are still inconclusive. Multiple studies didn't reveal a more unfavourable clinical outcome in hospitalized patients with HIV and COVID-19 compared to the general population. One factor which was proven to negatively influence the mortality in patients with HIV and SARS-CoV-2 is pulmonary tuberculosis. Other risks factors associated with more severe outcomes were the ones previously proven to worsen the prognosis of COVID-19 patients (advanced age, cardiovascular diseases, diabetes mellitus, chronic pulmonary disease, obesity etc.) (12-17).

\section{CONCLUSIONS}

The particularities of this case consist in the following: the patient was simultaneously diagnosed with HIV, SARS-CoV-2 and Kaposi sarcoma; he was a late presenter (CD4+ T cells $<350 / \mathrm{mm}^{3}$ ); he was identified with an AIDS defining disease; he developed a severe form of disseminated Kaposi sarcoma (with cutaneous, mucous and visceral involvement) 
and COVID-19 with acute respiratory insufficiency; he was a MSM patient.

We want to emphasize the importance of HIV early diagnosis. This can easily be done through periodical screening of the individuals at higher risk of contracting the virus. Also, as physicians, we should

\section{REFERENCES}

1. McIntosh K. COVID-19: Clinical features. 2021. UpToDate. Available at: https://www.uptodate.com/contents/covid-19-clinical-features.

2. Collins LF, Moran CA, Oliver NT, Moanna A, Lahiri CD, Colasanti JA, Kelley CF, Nguyen ML, Marconi VC, Armstrong WS, Ofotokun I, Sheth AN. Clinical characteristics, comorbidities and outcomes among persons with HIV hospitalized with coronavirus disease 2019 in Atlanta, Georgia. AIDS. 2020 Oct 1;34(12):1789-1794.

3. Dandachi D, Geiger G, Montgomery MW, Karmen-Tuohy S, et al.; HIV-COVID-19 consortium. Characteristics, Comorbidities, and Outcomes in a Multicenter Registry of Patients with HIV and Coronavirus Disease-19. Clin Infect Dis. 2020 Sep 9:ciaa1339.

4. Kasper DL. Fauci AS. Harrison Boli Infecțioase - Ediția a II-a. Editura All, 2020.

5. Flaherty KR. Pulmonary involvement in AIDS-related Kaposi sarcoma. 2019. UpToDate. Available at: https://www.uptodate.com/ contents/pulmonary-involvement-in-aids-related-kaposi-sarcoma.

6. Kanne JP, Yandow DR, Meyer CA. Pneumocystis jiroveci Pneumonia: High-Resolution CT Findings in Patients With and Without HIV Infection. American Journal of Roentgenology. 2012;198:W555-W561.

7. Fajgenbaum DC. HHV-8-associated multicentric Castleman disease. 2020. UpToDate. Available at: https://www.uptodate.com/contents/ hhv-8-associated-multicentric-castleman-disease.

8. Lugović L, Pusić J, Situm M, Buljan M, Bulat V, Sebetić K, Soldo-Belić A. Acroangiodermatitis (pseudo-Kaposi sarcoma): three case reports. Acta Dermatovenerol Croat. 2007;15(3):152-7.

9. Sunil M, Reid E, Lechowicz MJ. Update on HHV-8-associated malignancies. Curr Infect Dis Rep. 2010;12:147-54. be able to recognize the early signs KS in our patients so that to avoid the development of advanced forms and severe complications. All those cases require an interdisciplinary approach in order to manage the disease progression and to institute a proper treatment plan.

\section{Conflict of interest: none declared} Financial support: none declared

10. Rabkin CS. AIDS and cancer in the era of highly active antiretroviral therapy (HAART). Eur J Cancer. 2001;37:1316-9.

11. Bower M, Nelson M, Young AM, Thirlwell C, Newsom-Davis T, Mandalia S, et al. Immune reconstitution inflammatory syndrome associated with Kaposi's sarcoma. J Clin Oncol. 2005 Aug 1;23(22):5224-8.

12. Vally F, Selvaraj WMP, Ngalamika O. Admitted AIDS-associated Kaposi sarcoma patients: Indications for admission and predictors of mortality. Medicine (Baltimore). 2020 Sep 25;99(39):e22415.

13. Zhu F, Cao Y, Xu S, Zhou M. Co-infection of SARS-CoV-2 and HIV in a patient in Wuhan City, China. J Med Virol. 2020 Jun; 92(6):529-530.

14. Guo W, Ming F, Dong Y, et al. A Survey for COVID-19 among HIV/ AIDS Patients in Two Districts of Wuhan, China. Preprint research paper, The Lancet, 2020.

15. Gudipati S, Brar I, Murray S, McKinnon JE, Yared N, Markowitz N. Descriptive Analysis of Patients Living With HIV Affected by COVID-19. J Acquir Immune Defic Syndr. 2020 Oct 1;85(2):123-126.

16. Mirzaei H, McFarland W, Karamouzian M, Sharifi H. COVID-19 Among People Living with HIV: A Systematic Review. AIDS Behav. 2021 Jan;25(1):85-92.

17. Boulle A, Davies MA, Hussey H, Ismail M, Morden E, et al. Risk factors for COVID-19 death in a population cohort study from the Western Cape Province, South Africa. Clin Infect Dis. 2020 Aug 29:ciaa1198. 